\title{
Random Distribution of Long-Term Indicators of Variable Emission Conditions
}

\author{
W. BATKO ${ }^{a, *}$ AND B. PRZYSUCHA ${ }^{b}$ \\ ${ }^{a}$ Faculty of Mechanical Engineering and Robotics, Department of Mechanics and Vibroacoustics \\ AGH University of Science and Technology, al. A. Mickiewicza 30, 30-059 Kraków, Poland \\ ${ }^{b}$ Faculty of Management, Department of Quantitative Methods in Management of Economy \\ Lublin University of Technology, Nadbystrzycka 38, 20-618 Lublin, Poland
}

This study is dedicated to the problem of estimating uncertainties of long-term noise indicators, when differences in the sound level emission at various time periods of the calendar year are taken into consideration. This task is defined by referring their influence values - in the determined time intervals - to the year period. Due to the limited possibilities of a total monitoring of parameters necessary for the precise estimation of the long-term sound levels, this estimation process is often limited (in accordance with the EU environmental recommendations) to two condition classes. They are defined by two sound levels occurring with probabilities (frequencies) $p$ and $1-p$, in the analyzed reference period. In this paper we present a method of calculating uncertainties of this procedure assuming that frequency of determined events are known. The probability distribution for the estimated value was assessed. The developed model formalism of the estimation of uncertainties of long-term sound levels together with algorithms assigned to it, was analyzed. The proposed solution was illustrated by examples of uncertainty calculations of the averaged sound levels in acoustic assessments of environmental hazards.

PACS: $43.28 . \mathrm{Lv}, 43.50 .-\mathrm{x}, 02.50 . \mathrm{Cw}$

\section{Introduction}

One of the main issues while assessing the acoustic hazards is estimation of the average sound levels $L_{L T}^{(j)}$ in various times of the day: $j=1$, evening: $j=2$, night: $j=3$ in the entire calendar year.

In accordance with the Standard PN-ISO 1996-2:1999 they are defined by the formula:

$$
L_{A e q, L T}^{(j)}=10 \log \left(\sum_{i=1}^{N} 10_{i}^{0.1\left(L_{A e q, T}\right)}\right),
$$

where $N$ - number of samples for the reference time interval $T^{(j)}$ of the analyzed time of the: day $j=1$, evening $j=2$ and $j=3$ night, of the considered calendar day, $\left(L_{A e q, T}\right)_{i}$ - equivalent sound level $A[\mathrm{~dB}]$ for the $i$-th sample in the considered reference time period $T^{(j)}$.

Long-term average equivalent sound levels can be determined by various methods including calculating and measuring while taking into the account:

- Numerous input parameters characterizing noise emissions (from the noise sources) on the analyzed sites, including knowledge of their acoustic power levels as well as the equivalent sound levels - for the periods of their characteristic activities in the calendar year;

\footnotetext{
* corresponding author; e-mail: batko@uci.agh.edu.pl
}

- Variables, describing conditions of sound propagation for analyzed sites;

- Conditions of measurements in the analyzed localizations.

Their values constitute the input database for creating acoustic maps of the analyzed places. They are one of the information layers in the municipal System of Site Information supporting the environment management. They function within the Geographic Information System (GIS) and are explicitly related to geographic coordinates of the analyzed site.

Following the European Commission recommendations and domestic guidelines for preparing acoustic maps [1] in the case when there are no complete data for their calculations (until the precise obtaining of data will be possible) - the calculations of the long-term average noise indicators can be simplified to the estimation of equivalent sound levels of the basic noise sources, which are forming them. Conditions of their activity, determined by a percentage contribution of the noise emission in the calendar year, should be taken into consideration.

This taking into consideration leads to the dependence

$$
\begin{aligned}
& L_{L T}^{(j)}=10 \log \left(p 10^{0.1 L_{A}^{(j)}}+(1-p) 10^{0.1 L_{B}^{(j)}}\right), \\
& \quad j=1,2,3,
\end{aligned}
$$

where $p$ is the calendar year frequency of conditions occurring within the calendar year, determined by the noise 
level emission of the source $L_{A}^{(j)}$ while $1-p$ corresponds to the level $L_{B}^{(j)}$ occurring in the relevant time interval.

An activity variable of the considered sound source can be related to different excitations:

- Percentage fraction of workdays of noise source emission level $L_{A}^{(j)}$ in the calendar year versus the percentage fraction of days in which it is limited to the level $L_{B}^{(j)}$;

- Probability $p$ of an occurrence (in the calendar year) of atmospheric conditions favoring the determined noise emission (e.g. wind, temperature, humidity, or pressure) for the considered noise source $L_{A}^{(j)}$ versus the probability $1-p$ in which the noise emission is on the level $L_{B}^{(j)}$;

- Percentage fraction of heavy vehicles in the traffic structure, creating emission conditions of the analyzed noise source in the whole year;

- Probability $p$ of a source emission time $L_{B}^{(j)}$ in the calendar year, discussed at the background of frequency $1-p$ of occurring conditions characteristic for the acoustic background level $L_{B}^{(j)}$ on the considered site, etc.

Such estimations of the long-term average sound levels $L_{L T}^{(j)}$, in relation to the considered noise emission conditions in the analyzed sites should be supplemented by their uncertainty assessment at taking into account the randomness of the sound levels measurement results $L_{A}$ and $L_{B}$. According to the rules given in the Guide to the Expression of Uncertainty in Measurement issued by the International Standardization Organization (ISO) [2] recommended for applications in: Measure Service, accredited laboratories and other institutes of technical inspections and control, uncertainty of the estimated quantity $U(x)$ expressed by the formula

$$
U(x)=k(\alpha) u_{\mathrm{c}}(x)
$$

is determined by the standard deviation $s(x)$, of the observation $x_{i} ; i=1,2, \ldots, n$ observations multiplied by the coverage factor $k(\alpha)$ and combined standard uncertainty $u_{\mathrm{c}}$, being the probability distribution quantile of the considered control variable $x$, calculated for the significance level $\alpha$ of making an error in the assessment.

Realization of such procedure in relation to long-term average noise indicators $L_{L T}^{(j)}$ requires the knowledge of the distribution of the analyzed random variable $x=L_{L T}^{(j)}$.

In the sequel, our attention will be focused on assessing such distribution. The method of calculating of uncertainties of the procedure determined by relation (2), when knowing the occurrence frequency of the determined event creating the long-term average noise level in the calendar year will be presented.
The developed model formalism of the uncertainty assessment of long-term sound levels together with the algorithm assigned to it - was analyzed. The proposed solution was illustrated by examples of uncertainty calculations of averaged sound levels in acoustic estimations of environmental hazards.

\section{Procedure of the determination of the long-term average noise indicators}

As mentioned earlier in the paper, the determination of uncertainties of long-term average noise indicators, on the grounds of correction of the characteristic sound levels values creating acoustic hazards in the given site, in relation e.g. to atmospheric conditions, requires the determination of the random variable probability distribution $L_{L T}$ formula (2).

Its level is determined on the grounds of estimating influences of characteristic conditions of noise emission in the whole calendar year. They are brought to the determination of the occurrence frequency $p$ of the determined noise emission in the group of random events of levels $L_{A}$ and $L_{B}$. Frequency $p=\frac{t_{1}}{t_{1}+t_{2}}$ is determined by the exposition time $t_{1}$ of source $A$ considered within the group of conditions favoring the propagation of the acoustic wave of level $L_{A}$, and time $t_{2}$ in which the exposition of the noise level $L_{B}$ takes place.

When $p=\frac{1}{2}$, we have received logarithmic mean sound level. Procedure of determination probability distribution of the logarithmic mean $n$-sound levels is described in the publication [3].

It can be assumed that random variables $L_{A}$ and $L_{B}$ are independent, and their probability density function are $\rho_{L_{A}}(\cdot), \rho_{L_{B}}(\cdot)$ together with their distribution functions $\Psi_{L_{A}}(\cdot), \Psi_{L_{B}}(\cdot)$. It should be recalled that $L_{A}$ is determined in the interval $[a, b]$, while $L_{B}$ in the interval $[c, d]$, where numbers $a, b, c, d>0$.

It is assumed that the characteristic activity $p \in[0,1]$ (frequency of occurrence) of random variables is known $X, Y:\left[X=p 10^{0.1 L_{A}}, Y=(1-p) 10^{0.1 L_{B}}\right]$. They are independent due to the independence of variables $L_{A}, L_{B}$.

In order to determine the random variable distribution (3) it is necessary to determine the distribution of random variables $X, Y$.

Using the notation above we easily obtain the following relationship:

$$
\begin{aligned}
& \Psi_{X}(s)=P(X<s)=P\left(p 10^{0.1 L_{A}}<s\right) \\
& \quad=P\left(L_{A}<10 \log \left(\frac{s}{p}\right)\right)=\Psi_{L_{A}}\left(10 \log \left(\frac{s}{p}\right)\right) .
\end{aligned}
$$

From the relation (4) we obtain

$$
\Psi_{X}(s)=\Psi_{L_{A}}\left(10 \log \left(\frac{s}{p}\right)\right) .
$$

After calculating the derivative of the distribution function $L_{A}$ we obtain the equation for the destiny function 


$$
\begin{aligned}
& \rho_{X}(s)=\frac{\mathrm{d}\left[\Psi_{X}(s)\right]}{\mathrm{d} s}=\frac{\mathrm{d}\left[\Psi_{X}\left(10 \log \left(\frac{s}{p}\right)\right)\right]}{\mathrm{d} s} \\
& =\rho_{L_{A}}\left(10 \log \left(\frac{s}{p}\right)\right) \frac{\mathrm{d}\left[10 \log \left(\frac{s}{p}\right)\right]}{\mathrm{d} s} \\
& =\frac{10}{s \ln 10} \rho_{L_{A}}\left(10 \log \left(\frac{s}{p}\right)\right), \\
& s \in\left[p 10^{0.1 a}, p 10^{0.1 b}\right]
\end{aligned}
$$

By a similar procedure the distribution and the density functions for the variable $Y$ can be determined

$$
\Psi_{Y}(s)=\Psi_{L_{B}}\left(10 \log \left(\frac{s}{1-p}\right)\right)
$$

and

$$
\begin{gathered}
\rho_{Y}(s)=\frac{10}{s \ln 10} \rho\left(10 \log \left(\frac{s}{1-p}\right)\right), \\
s \in\left[(1-p) 10^{0.1 c},(1-p) 10^{0.1 d}\right] .
\end{gathered}
$$

Distribution of the random variable sum $X+Y$ can be determined by means of the probability distribution convolution $[4,5]$.

Denote via $\rho_{X+Y}(\cdot)$ the destiny of the variable $X+Y$. Then

$$
\rho_{X+Y}(z)=\rho_{X} * \rho_{Y}(z)=\int_{-\infty}^{z} \rho_{X}(x) \rho_{Y}(z-x) \mathrm{d} x .
$$

Substituting (5) and (6) into (7) the following is obtained:

$$
\begin{gathered}
\rho_{X+Y}(z)=\left(\frac{10}{\ln 10}\right)^{2} \int_{p 10^{0.1 a}}^{z-(1-p) 10^{0.1 c}} \frac{1}{x(z-x)} \rho_{L_{A}} \\
\times\left(10 \log \left(\frac{x}{p}\right)\right) \rho_{L_{B}}\left(10 \log \left(\frac{z-x}{1-p}\right)\right) \mathrm{d} x .
\end{gathered}
$$

The next step is the probability distribution calculation

$$
\begin{aligned}
& L_{L T}=10 \log (X+Y) \\
& \rho_{L_{L T}}(z)=\frac{\mathrm{d}\left[P\left(L_{L T}<z\right)\right]}{\mathrm{d} z} \\
& \quad=\frac{\mathrm{d}[P(10 \log (X+Y)<z)]}{\mathrm{d} z} \\
& =\frac{\mathrm{d}\left[P\left(X+Y<10^{0.1 z}\right)\right]}{\mathrm{d} z}=\frac{\mathrm{d}\left[\Psi_{X+Y}\left(10^{0.1 z}\right)\right]}{\mathrm{d} z} \\
& =\rho_{X+Y}\left(10^{0.1 z}\right) \frac{\mathrm{d}\left[10^{0.1 z}\right]}{\mathrm{d} z} \\
& =\frac{\ln 10}{10} 10^{0.1 z} \rho_{X+Y}\left(10^{0.1 z}\right) .
\end{aligned}
$$

Finally from the relation (9) we obtain

$$
\rho_{L_{L T}}(z)=\frac{\ln 10}{10} \rho_{X+Y}\left(10^{0.1 z}\right) .
$$

Inserting (8) to (10) we get

$$
\begin{aligned}
& \rho_{L_{L T}}(z)=\frac{10}{\ln 10} \int_{p 10^{0.1 a}}^{10^{0.1 z}-(1-p) 10^{0.1 c}} \frac{10^{0.1 z}}{x\left(10^{0.1 z}-x\right)} \rho_{L_{A}} \\
& \quad \times\left(10 \log \left(\frac{x}{p}\right)\right) \rho_{L_{B}}\left(10 \log \left(\frac{10^{0.1 z}-x}{1-p}\right)\right) \mathrm{d} x \\
& s \in\left[z_{0}, z_{1}\right]
\end{aligned}
$$

where the endpoints of the determination ability interval of the variable $L_{L T}$ are given by

$$
\begin{aligned}
& z_{0}=p 10^{0.1 a}+(1-p) 10^{0.1 c} \\
& z_{1}=p 10^{0.1 b}+(1-p) 10^{0.1 d} .
\end{aligned}
$$

Equation (11) allows calculating the probability distribution of the long-term average noise indicator given by Eq. (2). This, in turn, leads to calculations of expected value and variance as

$$
\begin{aligned}
& \mathrm{E} L_{L T}=\int_{z_{0}}^{z_{1}} z \rho_{L_{L T}}(z) \mathrm{d} z, \\
& \mathrm{E}\left(L_{L T}\right)^{2}=\int_{z_{0}}^{z_{1}} z^{2} \rho_{L_{L T}}(z) \mathrm{d} z, \\
& \operatorname{Var}\left(L_{L T}\right)=\mathrm{E}\left(L_{L T}\right)^{2}-\left(\mathrm{E} L_{L T}\right)^{2} .
\end{aligned}
$$

\section{Example}

In order to illustrate the work of the algorithm of the probability distribution estimation of the long-term average noise indicators $L_{L T}$ functions, the knowledge of sound levels distributions $L_{A}$ and $L_{B}$ was assumed. It was assumed that distribution is Gaussian trimmed to the relevant interval. This hypothetical assumption closely corresponds to the widely accepted assumption of normality of such observations. However, in the further analysis it should be taken into account that experimental investigations indicate (see [6]) that is reasonable to use other distributions that are not symmetric, left-skewed with the kurtosis significantly different than zero.

In the case when the random variable $X$ is of the Gaussian trimmed distribution on the interval $[a, b]$ with the expected value $\mu \in[a, b]$ and the standard deviation $\sigma$, its probability distribution is as follows:

$$
\rho_{X}(x)=\left\{\begin{aligned}
\frac{1}{F_{X}(b)-F_{X}(a)} \frac{1}{\sqrt{2 \pi} \sigma} & \exp \left(-\frac{(x-\mu)^{2}}{2 \sigma^{2}}\right), \\
& a \leq x \leq b, \\
0, & x \notin[a, b],
\end{aligned}\right.
$$

where

$$
F_{X}(x)=\int_{-\infty}^{x} \frac{1}{\sqrt{2 \pi} \sigma} \exp \left(-\frac{(t-\mu)^{2}}{2 \sigma^{2}}\right) \mathrm{d} t .
$$

Let us mark such distribution by $X \sim N_{[a, b]}(\mu, \sigma)$. 
Analysis of theoretical properties of the probability density function of the long-term average noise indicators was performed for random variables $L_{A} \sim$ $N_{[55,75]}(65,1.5)$ and the $L_{B} \sim N_{[45,65]}(58,1.5)$. Below, we show graphs of the random variables distribution, for various values of the parameter $p$.

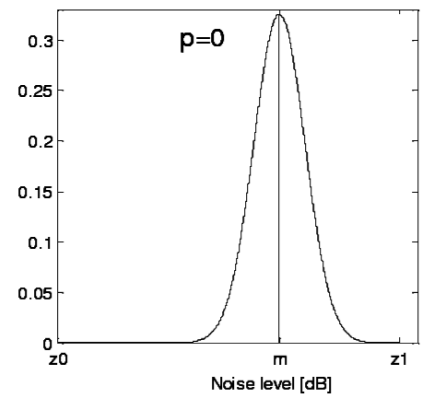

Fig. 1. Density function of $L_{L T}$ for $p=0$.

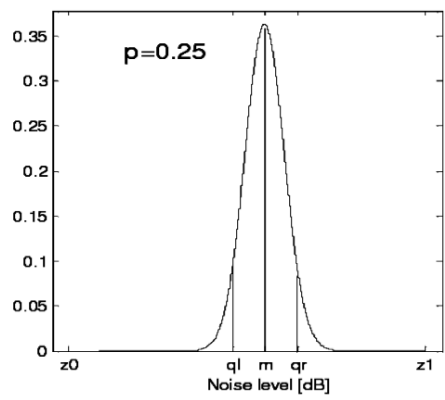

Fig. 2. Density function of $L_{L T}$ for $p=0.25$.

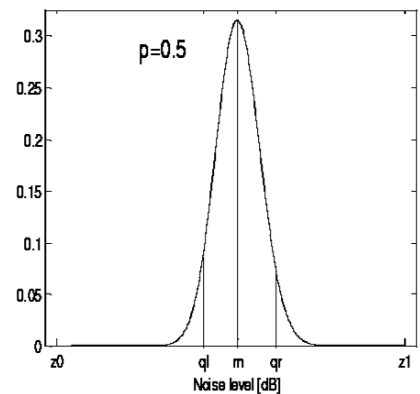

Fig. 3. Density function of $L_{L T}$ for $p=0.5$.

Calculation rules of the density probability function of the long-term average noise indicator proposed in paragraph 2 and based on random variable $L_{L T}=$ $10 \log \left(p 10^{0.1 L_{A}}+(1-p) 10^{L_{B}}\right)$ were constructed by means of the convex combination of random variables $10^{L_{A}}$ and $10^{0.1 L_{B}}$ describing the energy distribution of sound levels $L_{A}$ and $L_{B}$. In discussed experiments $n=2000$ results of control variables $L_{A}$ and $L_{B}$ were generated. To determine the density functions we have used the numerical integration methods - the Simpson method and

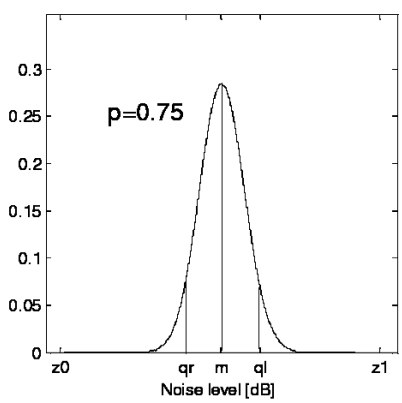

Fig. 4. Density function of $L_{L T}$ for $p=0.75$.

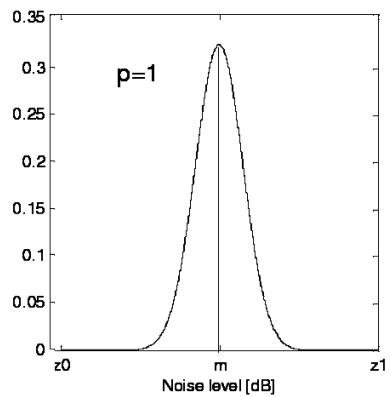

Fig. 5. Density function of $L_{L T}$ for $p=1$.

trapezoids method. Results of calculations are shown in Table, and shapes of density functions are illustrated in diagrams of Figs. $1-5$. In cases when $p=0$ or $p=1$ we are dealing with extreme distributions, i.e. when $p=0$ then $L_{L T}=L_{B}$ and when $p=1$ then $L_{L T}=L_{A}$.

It is seen in the diagrams that when $p$ varies within the interval $(0,1)$ distribution characteristics are also changing, which means that percentage contributions of each described effect is changing in the similar fashion. The expected values are marked in graphs by perpendicular lines in the middle; determination intervals - extreme lines; quantiles left (0.05) and right (0.95) — inside lines. It is worth to noting that the expected value of the vari-

Results of calculations.

TABLE

\begin{tabular}{|c|c|c|c|c|c|}
\hline & $P=0$ & $P=0.25$ & $P=0.5$ & $P=0.75$ & $P=1$ \\
\hline $\begin{array}{l}\text { beginning } \\
\text { of the } \\
\text { interval } z 0\end{array}$ & 45 & 50.1188 & 52.4036 & 53.8930 & 55 \\
\hline $\begin{array}{l}\text { left } \\
\text { quantile } \\
(0.05) q l\end{array}$ & & 59.3588 & 60.8336 & 61.8030 & \\
\hline $\begin{array}{l}\text { expected } \\
\text { value } M\end{array}$ & 58 & 61.1354 & 62.8523 & 64.0562 & 65 \\
\hline $\begin{array}{l}\text { right } \\
\text { quantile } \\
(0.95) q r\end{array}$ & & 62.9888 & 64.9936 & 66.4130 & \\
\hline $\begin{array}{l}\text { end of the } \\
\text { interval } z 1\end{array}$ & 65 & 70.1188 & 72.4036 & 73.8930 & 75 \\
\hline $\begin{array}{l}\text { standard } \\
\text { deviation } s\end{array}$ & 1.5 & 1.1027 & 1.2671 & 1.4003 & 1.5 \\
\hline
\end{tabular}


able $L_{L T}$ is changing from the expected value of variable $L_{B}: \mu_{B}=58$ for $p=0$ to the expected value of variable $L_{A}: \mu_{A}=65$ : for $p=1 \mu_{L_{L T}} \in[58,65]$. The confidence intervals and standard deviations behave in a similar way.

\section{Summary and conclusion}

Important result of this paper is to sketch out method of determination of the algorithm of estimation of the probability density function for the long-term noise indicators, estimated by simplified method measurement-calculation, when knowing characteristic activity periods of the source creating the acoustic state in the analyzed environmental site.

This is a new, strict approach to estimation uncertainties of long-term average noise indicators, for the customarily procedure in the consideration of control of acoustic environment. In contrast to existing rules for the evaluation of uncertainty recommended in guide [2], the essence of this approach is to assume the propagations principle as the basis for the calculation uncertainties of outcome control and to give the possibility to skip over a series of troubling assumption used so far in practice.

Formulated algorithm is the universal tool for estimating uncertainties of the analyzed noise indicators. The possibility of the proper uncertainty assessment for an arbitrary form of the sound level probability distribution of the source and of the acoustic background deserves attention.

Development of proposed method will be concerned on a case when the $p$ percentage fraction of participation weekdays noise emission of source $L_{A}^{(j)}$ in the calendar year to the $1-p$ percentage fraction days whose emission is limited to the level $L_{B}^{(j)}$, will be random variable.

The results of the simulation numerical experiments indicated asymmetry of the density distribution function for the long-term average sound levels. Thus, it suggests the necessity of a very cautious use of the so far applied methods of assessments of standard uncertainties of controlled noise indicators. These methods base on generally recommended guidelines contained in the publication Guide to the Expression of Uncertainty in Measurement [2], which assume the normality of the observation distribution form, which is not justified by the control practice [6].

To summarize considerations performed in this paper, it can be stated that the presented modus operandi at the estimation of the long-term noise indicators does not create any limitations and - in future - will be more broadly applied in various control algorithms. It can also serve as the reference base for the correctness assessment of recently used estimation methods. It can supplement them in several analyses.

\section{Acknowledgments}

The study was performed within the framework of the project: N R03 0030 06/2009.

\section{References}

[1] R. Kucharski, Guidelines of Elaborate of Acoustic Maps, Publication of Chief Inspectorate of Environmental Protection, Warsaw 2011.

[2] Guide to the Expression of Uncertainty Measurement, International Organization for Standardization, BIPM, IEC, ISO, ISBN 92-67-10188-9, SO/IEC Guide 98-3:2008.

[3] W. Batko, B. Przysucha, Arch. Acoust. 35, 543 (2010).

[4] P. Billingsley, Probability and Measure, 2nd ed., Wiley, New York 1986, p. 264.

[5] T. Gersternkorn, T. Śódka, Theory of Combination and Probability, PWN, Warsaw 1983, p. 292.

[6] W. Batko, B. Stępień, in: Proc. 35th Winter School on Vibroacoustical Hazards Suppressions, Wista (Poland), Ed. M. Roczniak, Upper Silesian Division of the Polish Acoustical Society, Gliwice 2007, p. 5. 